\title{
Introduction to Emerging Topics in Digital Government Minitrack
}

\author{
Theresa Pardo \\ University at Albany - SUNY \\ tpardo@ctg.albany.edu
}

\author{
Elin Whiborg \\ Linkoping University, Sweden \\ elin.wihlborg@liu.se
}

\author{
Robert Krimmer \\ Tallinn University of Technology \\ robert.krimmer@ttu.ee
}

The Digital Government Emerging Topics MiniTrack provides a home for incubating new topics and trends in Digital Government research. Digital Government as an academic field is evolving; new directions of research and practice are emerging while others are becoming accepted as foundational. These developments take place at the crossroads of different academic disciplines and in close connection to the practices in governments around the globe. This minitrack invites papers positioned in relation to emerging issues and challenges in digital government including changing or emerging needs and trends. Submissions must speak specifically to the emerging nature of the topic and how the research presented builds new understanding by relating the research to the central developments in the field of digital government.
Topics and research areas include but are not limited to:

- Conceptual and practice-based boundariesAgendas for Digital Government research

- Ethics of Digital Government from theoretical and practical views

- Digital Government education and competences

- ICT-enabled policy making and Digital Government policy

- IT, government, and the diversity of population and citizens

- Participatory approaches in government, for instance co-production

- Trust in and safe use of Digital Government

- Comparative studies focused on emerging issues or opportunities in the field of Digital Government

- Theories and models to analyze e-government 